\title{
COMPORTAMENTO TERRITORIAL EM AVES: REGULAÇÃO POPULACIONAL, CUSTOS E BENEFÍCIOS
}

\author{
Vanessa Cardoso Tomaz ${ }^{1,2} \&$ Maria Alice S. Alves ${ }^{2}$ \\ ${ }^{1}$ Programa de Pós-graduação em Ecologia, Universidade Federal do Rio de Janeiro (UFRJ). Caixa Postal: 68.020, CEP: 21.941-540. Ilha do Fundão, \\ Rio de Janeiro, RJ. \\ ${ }^{2}$ Departamento de Ecologia, Universidade do Estado do Rio de Janeiro, Instituto de Biologia Roberto Alcantara Gomes. Rua São Francisco Xavier, \\ 524. Cep 20550-011. Rio de Janeiro, RJ. \\ E-mails: v.c.tomaz@gmail.com, masaal@globo.com.
}

\section{RESUMO}

Diversas hipóteses têm sido sugeridas para explicar o comportamento territorial das aves, a maioria delas associadas com a obtenção de alimento, de parceiros sexuais e de local adequado para a nidificação. No presente artigo abordamos fatores relacionados ao comportamento territorial que interferem na dinâmica populacional e no sucesso reprodutivo de aves. Discutimos benefícios e custos da territorialidade, incluindo as interações entre indivíduos territoriais e flutuantes. Além disto, indicamos a importância das informações sobre territorialidade na elaboração de programas de conservação para espécies de aves ameaçadas.

Palavras-chave: Territórios, custos-benefícios, modelos de territorialidade, indivíduos flutuantes.

\section{ABSTRACT}

TERRITORIAL BEHAVIOR IN BIRDS: POPULATION REGULATION, COSTS AND BENEFITS. Several hypotheses have been suggested to explain territorial behavior in birds population regulation, costs and benefits. The majority of them are associated with finding food, sexual partners and appropriate breeding sites. In the present paper we describe factors related to territorial behavior that interfere with population dynamics and breeding success in birds. We discuss costs and benefits of territoriality, including interactions between territorial and floating individuals. We highlight the importance of knowledge about territorial behavior for the development of effective conservation programs of threatened bird species.

Keywords: Territories, costs-benefits, territoriality models, floater individuals.

\section{RESUMEN}

COMPORTAMIENTO TERRITORIAL EN AVES: REGULACIÓN POBLACIONAL, COSTOS Y BENEFICIOS. Diversas hipótesis han sido sugeridas para explicar el comportamiento territorial de las aves, la mayoría de ellas asociadas a la obtención de alimento, de parejas sexuales y de sitios de nidificación apropiados. En el presente trabajo abordamos los factores relacionados con el comportamiento territorial que afectan la dinámica poblacional y el éxito reproductivo de las aves. Discutimos los costos y beneficios de la territorialidad, incluidas las interacciones entre individuos territoriales y fluctuantes. Además, mostramos la importancia de la información sobre la territorialidad en el desarrollo de programas de conservación para especies de aves amenazadas.

Palabras clave: Territorios, costos-beneficios, modelos de territorialidad, individuos fluctuantes.

\section{INTRODUÇÃO}

A primeira publicação a mencionar a existência de territorialidade entre as aves data de 1622 (Nice 1941). $\mathrm{Na}$ ocasião, foi descrito o peculiar comportamento de machos do rouxinol Luscinia megarhyncha em impedir a aproximação de outros indivíduos da sua mesma espécie, exceto as fêmeas com as quais estavam pareados (Alexander 1936 apud Nice 1941).
O primeiro ornitólogo a detalhar a teoria por trás de tal comportamento foi Altum, em 1868 (Bock 2001). Segundo ele, o comportamento territorial tem a finalidade de manter uma área exclusiva para cada par reprodutor, a qual deve conter suprimento adequado de alimento para eles próprios e para sua prole. Neste sentido, o tamanho dos territórios é variável, estando relacionado com a produtividade do ambiente. Outra proposição importante e inovadora 
feita por Altum é de que a disputa entre os machos se dá pelos territórios e não pelas fêmeas (Mayr 1935 apud Nice 1941). Entretanto, Altum não alcançou projeção internacional e suas idéias ficaram restritas ao seu país de origem, a Alemanha (Bock 2001). Foi em 1920, com o britânico Howard, que o mundo finalmente começou a discutir a teoria que permeava o comportamento territorial das aves (Bibby 2003). Apesar de não ter lido o trabalho de Altum, a proposição de Howard foi essencialmente a mesma. Acrescentou ainda a idéia de que o comportamento territorial é um fator que limita a densidade de pares reprodutores (Howard 1920).

Posteriormente, diversas outras funções para o comportamento territorial foram sugeridas: evitar epidemias, assegurar a sincronização entre o período reprodutivo de machos e fêmeas, evitar cópulas extra-par por parte das fêmeas, proteger o par reprodutor e os filhotes de interferências externas, estimular a dispersão dos filhotes, reduzir a predação e assegurar um local adequado para nidificação (Allen 1934, Gibb 1956, Hinde 1956, Komdeur 1996, Dickinson 1997).

Parte da controvérsia acerca da territorialidade das aves se deve à subjetividade do termo 'território', que foi utilizado por diversos pesquisadores com sentidos diferentes, frequentemente não explicitados em suas publicações. Maher \& Lott (1995) citam 11 definições diferentes para territorialidade em trabalhos realizados com aves. Houve várias tentativas quanto à padronização da classificação e definição dos vários tipos de território (Nice 1941, Itô 1980). As categorias de territórios propostas vão desde o território clássico, onde há sobreposição total entre a área de vida e o território, até territórios dormitórios, que são restritos aos poleiros ou ocos de árvores em que os indivíduos dormem (Nice 1941).

A despeito de todos os aspectos considerados até então, o termo território pode ser definido, em sentido amplo, como uma área que é defendida por um indivíduo (ou mais de um indivíduo) em algum momento, seja através de ataque direto ou de intimidação por display visual e/ou vocalização (referências em Maher \& Lott 1995). Esta é a definição mais freqüente e parece ser também a mais conveniente para um conceito com tal carga de subjetividade, por ser extremamente simples e inclusiva. Uma definição ecológica (e não comportamental, como a previamente citada) está relacionada com a exclusividade no uso de determinado espaço (referências em Maher \& Lott 1995). Esta definição é especialmente útil em situações onde o comportamento não é registrado, como em estudos realizados com radio-tracking. Para evitar enganos, definições específicas, que contemplem não apenas o embasamento conceitual, como também operacional (como será realizado o registro empiricamente), devem ser adequadamente explicitadas nos trabalhos sobre territorialidade.

Metodologicamente, a definição dos limites de um território deve ser criteriosa, onde a marcação individual das populações estudadas é necessária. Uma coleção de pontos em que determinado indivíduo é registrado, mesmo que apenas durante o período reprodutivo, não deve ser interpretada, à priori, como sendo o seu território (Brown 1969), pois nem todos os territórios apresentam sobreposição total com a área de vida.

Outra questão importante, apontada por Davies (1941), está na detectabilidade do comportamento territorial pelo observador. Os ambientes tropicais, quando comparados aos temperados, apresentam uma tendência de aumento da diversidade de espécies acoplada com redução dos tamanhos populacionais. Segundo Davies, o tamanho reduzido das populações nos trópicos pode levar a uma situação em que não há estímulo para exibição de comportamento territorial. Este problema metodológico é acentuado em espécies que dependem do contato direto entre os indivíduos para exibição de comportamento territorial, o que raramente ocorre em populações pequenas. Ainda quanto à detectabilidade, deve-se considerar a possibilidade de o comportamento territorial ser mediado por alguma característica imperceptível ao observador, como a reflectância da luz UV. Este parece ser o caso dos machos de Cyanistes caeruleus, que respondem de forma mais intensa aos vizinhos que apresentam maiores valores de reflectância da luz UV do que aos que apresentam menores valores (reflectâncias manipuladas experimentalmente, Poesel et al. 2007).

Diversos são os métodos utilizados para traçar os limites dos territórios a partir de dados empíricos. O mais comum é o Mínimo Polígono Convexo (MPC) (Barg etal.2005). A principal vantagem do MPC éa sua simplicidade: os limites são traçados pela união entre os pontos de registro mais externos. A desvantagem é 
que não considera a configuração interna do território. Alternativas que oferecem estimativas mais precisas são baseadas na Distribuição de Utilização (DU) do espaço pelos indivíduos (Barg et al. 2005). A DU consiste na distribuição de frequências relativas de ocupação de um indivíduo entre as unidades de espaço (Van Winkle 1975).

\section{A TERRITORIALIDADE COMO REGULADORA DA DENSIDADE POPULACIONAL}

Um dos grandes questionamentos que logo surgiu sobre as conseqüências da territorialidade para as populações foi: o comportamento territorial regula a densidade de uma população? Para Lack (1954 apud Bibby 2003), a territorialidade não tem papel limitante na densidade de indivíduos que se reproduzem em uma população, ou seja, todos os indivíduos adultos que vivem em uma determinada localidade são capazes de estabelecer seus territórios e reproduzir. Isso implica em aceitar que os territórios possuem uma elasticidade ilimitada, podendo ser reduzidos tanto quanto for necessário para que todos os indivíduos possam se estabelecer. Entretanto, a combinação entre as evidências comportamentais (indivíduos que são impedidos de estabelecerem territórios em locais que pertencem ao território de outro indivíduo) e ecológicas (estabilidade na densidade populacional de indivíduos que se reproduzem e existência de indivíduos que, embora sexualmente maduros, não se reproduzem), apesar de não serem conclusivas, apontam para o lado oposto, sugerindo que a territorialidade atua na regulação da densidade de indivíduos que se reproduzem em uma população (Brown 1969, Newton 1992).

Brown (1969) propôs um modelo com três cenários distintos para a relação território-densidade (Figura 1), onde as consequiências da territorialidade foram medidas em termos de sucesso reprodutivo para cada nível de densidade. No nível 1 a densidade populacional é pequena, não há ocupação de habitats marginais e não existem indivíduos flutuantes (ou seja, que não possuem um território estabelecido), sendo possível que a densidade populacional aumente com a redução do tamanho dos territórios (não indefinidamente, mas até atingirem um limite mínimo). Neste nível, como não há competição por territórios, o comportamento territorial não desempenha um papel limitante da capacidade reprodutiva da população.

No nível 2 a ocupação dos habitats ótimos é máxima e alguns indivíduos passam a fixar seus territórios em habitats marginais; não existem indivíduos flutuantes. Neste nível a densidade de pares reprodutores nos habitats ótimos é constante, enquanto nos marginais ela é variável. Pode-se, portanto, concluir que a territorialidade neste nível apenas funciona como regulador do tamanho populacional para aquele grupo de indivíduos que estão estabelecidos nos habitats ótimos. Os pares reprodutores estabelecidos nos habitats ótimos, em comparação com aqueles estabelecidos nos habitats marginais, têm maior sucesso reprodutivo. Comparativamente com o nível 1 , no nível 2 as taxas de produção de filhotes por indivíduo são menores, entretanto o número absoluto de filhotes produzidos é maior.

No nível 3 a ocupação de todos os habitats disponíveis é máxima, logo a densidade de indivíduos que estão se reproduzindo alcançou o seu limite superior graças ao comportamento territorial. Os indivíduos que não conseguem estabelecer seus territórios não reproduzem, tornando-se indivíduos flutuantes. Quanto maior for a proporção de indivíduos flutuantes, menor vai ser a taxa de produção de filhotes por indivíduo, mas o número absoluto de filhotes produzidos é maior, quando comparado com o nível 2, e constante.

Diversas inconsistências foram encontradas entre dados empíricos e o modelo de Brown, principalmente por ser um modelo simples, que considera um número reduzido de variáveis e não incorpora as respostas individuais às variações na densidade populacional. Por exemplo, a redução do tamanho dos territórios, que já é observada no nível 1 do modelo, geralmente implica na redução do sucesso reprodutivo dos pares (Arcese $e t$ al. 1992, Paradis et al. 2002). Dessa forma, o aumento do número de pares reprodutores no nível 1 não acarreta necessariamente no aumento da capacidade reprodutiva em nível populacional. De forma semelhante, a predição de que, no nível 2, o sucesso reprodutivo dos pares estabelecidos nos habitats ótimos é maior do que o daqueles estabelecidos em habitats marginais pode ser subvertida em decorrência do efeito negativo da alta densidade dos habitats ótimos sobre os pares ali estabelecidos (Vickery 1992, Gilroy \& Sutherland 2007). Esse efeito é conseqüência, por exemplo, da atração exercida pela grande densidade populacional 


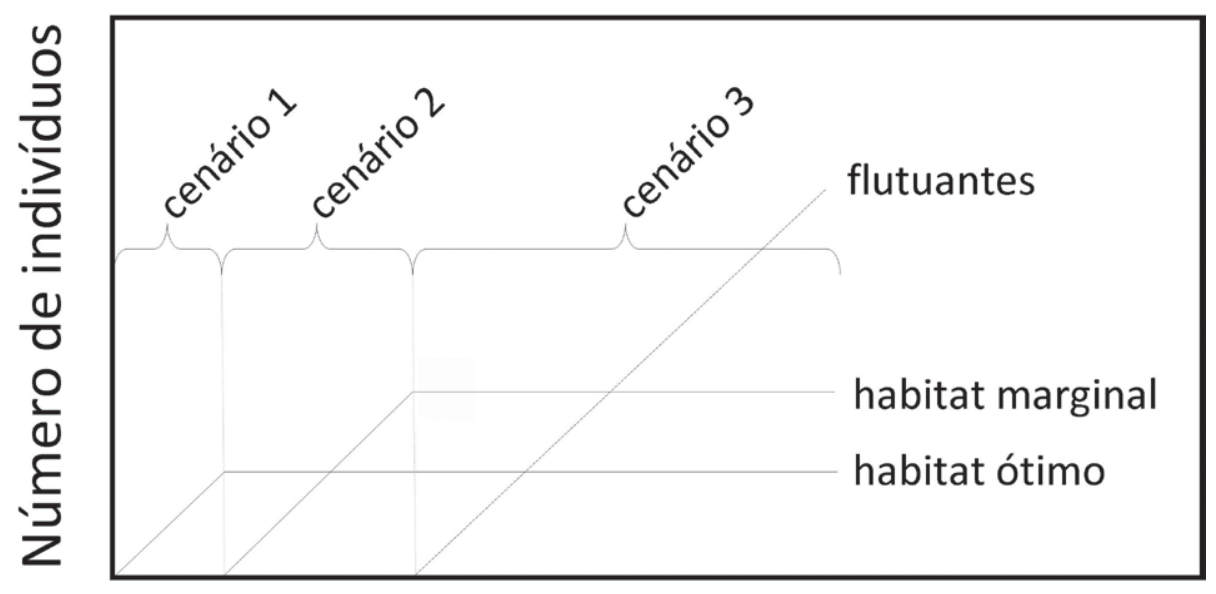

Tamanho da população

Figura 1. Representação gráfica do modelo de Brown (1969) para regulação da densidade populacional pelo comportamento territorial em aves. Adaptado de Heg et al. (2001).

Figure 1. Graphic representation of the model of Brown (1969) for populational density regulation by territorial behavior in birds. Adapted from Heg et al. (2001).

sobre os predadores e por uma maior disponibilidade de alimento nos habitats ótimos, atraindo os flutuantes (Dunn \& Tessaglia 1994, Carrete et al. 2006). Adicionalmente, considerando o cenário 2, a ausência de indivíduos flutuantes não é uma regra comprovada empiricamente. Trabalhos vêm mostrando que, dependendo da produtividade do ambiente em que a população considerada se encontra, manter-se como flutuante em habitats ótimos pode ser uma escolha com valor adaptativo, mesmo havendo a possibilidade de estabelecer território em habitat marginal (Komdeur 1996). Por fim, a constância no número de filhotes produzidos no cenário 3 frequentemente não é observada na natureza, em decorrência das interferências, conflitos e distúrbios diretos provocados pelos indivíduos flutuantes (Arcese et al. 1992, Jenkins \& Jackman 1993).

Um modelo matemático recente, de LópezSepulcre \& Kokko (2005), incorpora uma grande quantidade de variáveis para explicar a regulação do tamanho populacional pela territorialidade, integrando os custos e benefícios individuais e a dinâmica populacional (Figura 2). Duas possibilidades emergem deste modelo. A regulação do tipo 1 ocorre quando as taxas reprodutivas são elevadas, permitindo que territórios relativamente pequenos sejam capazes de produzir um número mediano de filhotes. Neste caso a densidade populacional é alta, os territórios mantêm um tamanho fixo e são pequenos. Assim, os indivíduos que não conseguem se estabelecer tornam-se flutuantes e a densidade da população passa a ser regulada pelo número de flutuantes presentes e sua capacidade de interferir nas taxas reprodutivas dos donos de território.

A regulação do tipo 2 ocorre quando a taxa reprodutiva é baixa, ou quando há pequena resposta da taxa reprodutiva em relação ao aumento do tamanho do território. Neste caso, as populações são relativamente pequenas e não existem indivíduos flutuantes. Os indivíduos recém-ingressos na fração reprodutiva da população conseguem estabelecer territórios prontamente, constringindo os territórios dos demais indivíduos até um ponto em que a natalidade se iguala à mortalidade. Assim, o tamanho da população é regulado exclusivamente pela interação entre donos de território.

Um parâmetro importante na definição do tipo de regulação é o custo da defesa territorial. Alto custo na defesa territorial leva a alta mortalidade entre os donos de território e, consequentemente, a uma maior taxa de substituição nesta fração da população. Se as taxas de substituição de donos de território são altas, a ocorrência de flutuantes se torna mais rara, favorecendo a regulação do tipo 2 (pela interação entre donos de território).

Os dois tipos de regulação podem existir em populações de uma mesma espécie. Por exemplo, tomando como parâmetro a qualidade do habitat (que permite traçar um paralelo entre o modelo do Brown e o de López-Sepulcre \& Kokko), a regulação do tipo 1 


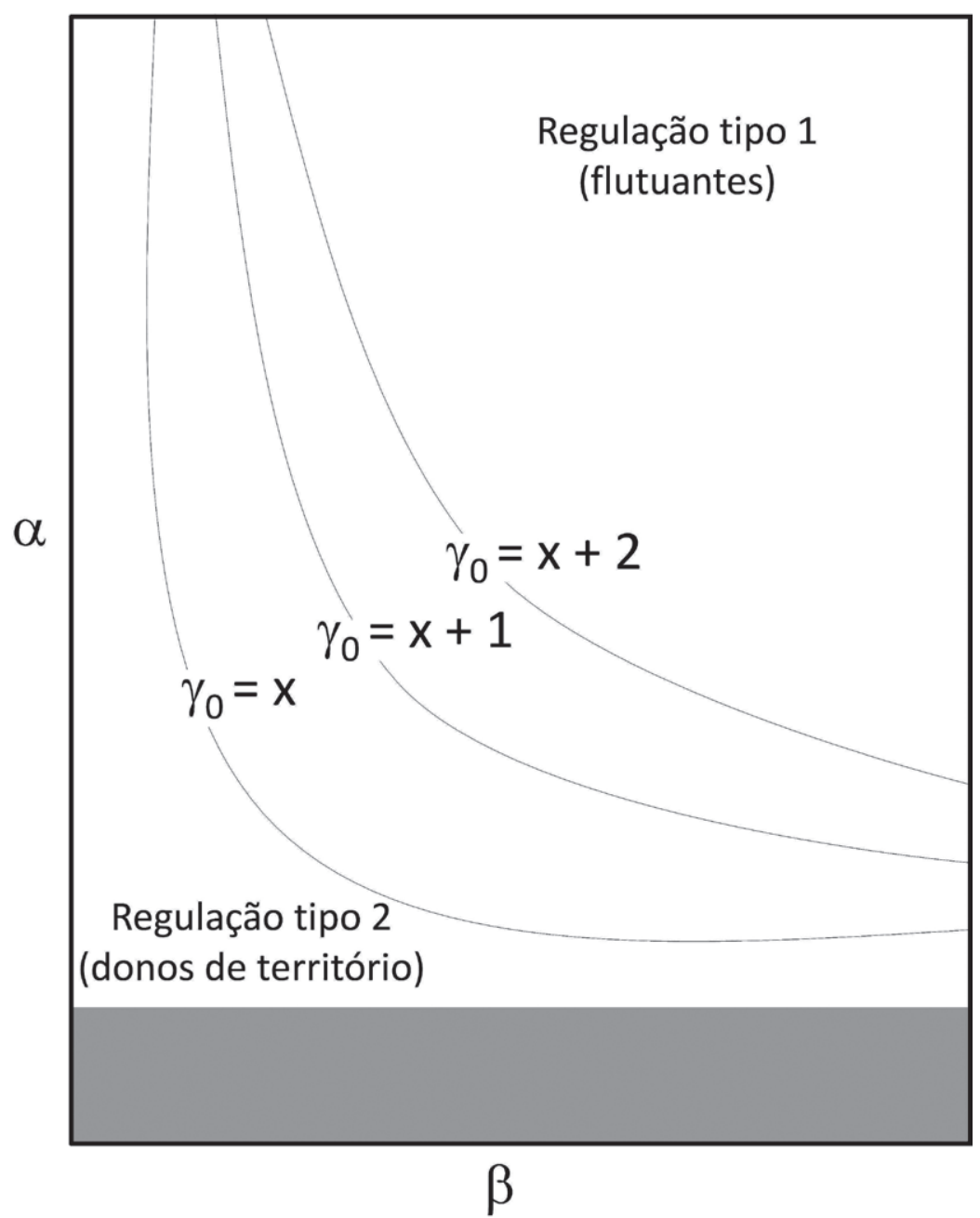

Figura 2. Representação gráfica do modelo de López-Sepulcre \& Kokko (2005) para regulação da densidade populacional pelo comportamento territorial. Os eixos $\alpha$ e $\beta$ estão relacionados com a capacidade reprodutiva da população (quanto maiores os valores de $\alpha$ e $\beta$,

maior a capacidade reprodutiva) e $\gamma_{0}$ representa os custos da defesa territorial. Para cada valor de $\gamma_{0}$, a área acima e à direita da curva indica regulação do tipo 1, enquanto as áreas abaixo e à esquerda indicam regulação do tipo 2. A área cinza do gráfico indica extinção da população. Adaptado de López-Sepulcre \& Kokko (2005).

Figure 2. Figure 1. Graphic representation of the model of López-Sepulcre \& Kokko (2005) for populational density regulation by territorial behavior. Adapted from Heg et al. (2001). Axes $\alpha$ and $\beta$ represent the reproductive capacity of the population (the greater their values, the greater is the reproductive capacity) and $\gamma_{0}$ represents the costs of territorial defense. For each value of $\gamma_{0}$ the curve area above and the right will stand for type 1 regulation, while the curve areas below and to the left will indicate type 2 regulation. The gray area represents elimination of that population. Adapted from López-Sepulcre \& Kokko (2005).

ocorreria em habitats ótimos, enquanto a regulação do tipo 2 em habitats marginais. Outra predição do modelo de López-Sepulcre \& Kokko (2005) é que as populações territoriais são menores quando comparadas a populações hipotéticas não territoriais. Isso significa que, na maior parte dos cenários considerados, a territorialidade beneficia apenas a fração da população que é capaz de se manter em status reprodutivo, sendo o efeito líquido sobre a população negativo. Esses autores sugerem ainda que a adição de mais variáveis ao modelo pode torná-lo mais realista, tais como eventos estocásticos, esforço na defesa territorial diferenciado entre os tipos de intrusos, variação na qualidade do habitat, variação no comportamento em resposta a diferentes densidades populacionais. Também é necessário aplicar as predições às populações reais para avaliar a sua validade.

É importante ainda ressaltar que, em determinadas situações, a densidade populacional pode não ter nenhuma relação com o tamanho e/ou número de territórios, por exemplo, quando existe uma quantidade limitada de sítios para nidificação. Neste caso, o número de pares reprodutivos capaz de estabelecer territórios e se reproduzir é equivalente ao número de sítios, independente da quantidade de indivíduos sexualmente maduros na população (Brush 1983). 


\section{QUANDO UMA AVE DEFENDE TERRITÓRIO?}

Brown (1964) propôs que um animal apenas estabelece um território quando os benefícios proporcionados por este são maiores do que os custos. Entre as vantagens de possuir um território podemos destacar a maior facilidade de acesso ao alimento, o aumento na chance de conseguir um parceiro para reprodução, a obtenção de um sítio apropriado para nidificação e a proteção da prole (Brown 1964, Adams 2001). Entre os custos estão o risco de sofrer algum ferimento nas disputas territoriais e o gasto de tempo e energia na defesa do território (Brown 1964, Adams 2001). Tanto os custos quanto as vantagens podem ser quantificados em termos de sucesso reprodutivo (quando o território está relacionado com a reprodução) e, mais genericamente, em termos energéticos (Gill \& Wolf 1975). O sucesso reprodutivo e/ou o saldo energético são dinâmicos, dependendo, por exemplo, do número de indivíduos flutuantes presentes, da taxa de mortalidade da população e da quantidade de energia disponível no ambiente (Gill \& Wolf 1975, Kokko et al. 2006). O papel de cada fator na decisão final (ser ou não ser territorial) é variável entre as espécies, entre as populações de uma mesma espécie (Fort \& Otter 2004) e até mesmo entre indivíduos de uma mesma população (Gill \& Wolf 1975). Neste sentido, são de extrema importância caracteres filogenéticos e genéticos.

Em geral, o gasto na defesa contra a invasão por indivíduos flutuantes é maior do que o gasto para contrabalançar a pressão exercida pelos territórios vizinhos (Myers et al. 1979). Alguns dos efeitos negativos gerados por indivíduos flutuantes sobre donos de territórios são: exploração de recursos presentes nos territórios, distúrbios ao ninho, cópulas extra-par e parasitismo de ninhada (Davies \& Houston 1981, Komdeur 1996, Sandell \& Diemer 1999, Kempenaers et al. 2001). Para a população de maneira geral, entretanto, a presença de indivíduos flutuantes pode ser positiva, uma vez que substituem os donos de territórios que morrem ou desertam (Newton 1992). O principal efeito negativo exercido por donos de territórios vizinhos são as cópulas extra-par (Kempenaers et al. 2001). A freqüência de paternidade extra-par creditada a donos de territórios vizinhos é, inclusive, muito superior àquela creditada a indivíduos flutuantes (por exemplo, 47\% e 13\%, respectivamente, dos filhotes produzidos por cópulas extra-par no estudo de Kempenaers et al. 2001 com a andorinha Tachycineta bicolor). Isso se deve, possivelmente, à recusa das fêmeas em copularem com os flutuantes, que são, geralmente, indivíduos de menor qualidade (Møller \& Ninni 1998).

Raramente um indivíduo flutuante tem sucesso em invadir e ocupar um território pré-estabelecido, expulsando o antigo dono (Hahn \& Bauer 2008). Isso porque, em geral, os donos de território são mais fortes e mais agressivos do que os flutuantes (Kokko et al. 2006), além de apresentarem plumagem mais conspícua, o que é um indicativo de seu status (Pryke \& Andersson 2003). Apenas em casos extremos insistem em desafiar um dono de território e geralmente o fazem em habitats ótimos (Heg et al. 2000). Isso ocorre, por exemplo, quando a expectativa de vida dos flutuantes é pequena e esses não têm outra opção para conseguir algum sucesso reprodutivo (Grafen 1987). Flutuantes também podem vir a desafiar repetidamente um dono de território sem a intenção imediata de expulsá-lo ou de utilizar recursos do território, mas sim de se familiarizar com a área e seus ocupantes (Bruinzeel \& Van de Pol 2004). Alternativamente, quando a população em questão apresenta alta taxa de mortalidade entre os donos de território, a estratégia mais vantajosa para os flutuantes pode ser simplesmente esperar pela morte de um deles (Kokko et al. 2006).

Neste contexto, quando a população em questão apresenta territórios com limites rígidos, um flutuante apenas consegue se estabelecer quando o dono de um território desaparece, deixando a área que ocupava disponível. Por outro lado, se a população apresentar territórios com limites relativamente flexíveis, então um indivíduo flutuante pode vir a estabelecer um território diminuto entre dois territórios pré-estabelecidos, expandindo seus limites posteriormente, quando já não é mais percebido como um flutuante, mas sim como vizinho, pelos donos de territórios adjacentes (Heg et al. 2000).

Considerando que os territórios disponíveis para uma população apresentam diferentes qualidades, um importante dilema se apresenta para flutuantes e donos de território: é mais vantajoso ocupar um território de baixa qualidade agora ou tentar estabelecer um território de alta qualidade no futuro, correndo o risco de morrer e/ou reduzir o sucesso reprodutivo antes de consegui-lo (Kokko \& Sutherland 1998, Sih \& Mateo 2001, Heg et al. 2003)? Em geral, as deserções são 
mais freqüentes entre os donos de territórios de baixa qualidade e as expulsões provocadas por flutuantes são mais freqüentes em territórios de alta qualidade (Heg et al. 2003, Blondel et al. 2000).

Para as fêmeas, os fatores que influenciam na escolha por ser ou não ser flutuante são diferentes. A possibilidade de parearem com um macho previamente pareado (tornando-se a segunda fêmea), em geral, é uma estratégia melhor do que se manter como flutuante, pois assim são capazes de conseguir algum sucesso reprodutivo, mesmo que pequeno (Johnson et al. 1994). Ou seja, como predito intuitivamente, a escolha se dá em função de alcançar o maior sucesso reprodutivo possível.

Quanto ao tamanho, os territórios são extremamente variáveis, refletindo a ação de alguns fatores, tais como qualidade do habitat, interação com donos de territórios vizinhos e indivíduos flutuantes, distribuição dos recursos, estágio reprodutivo e experiência (Adams 2001, Fort \& Otter 2004, Barg et al. 2005). O tamanho do território de um indivíduo pode, por exemplo, determinar o seu sistema de acasalamento (Davies 1992). É amplamente documentada uma relação inversa entre a densidade populacional e o tamanho dos territórios, e uma relação direta entre o tamanho dos territórios e o tamanho das ninhadas (Both \& Visser 2000). Essas relações, entretanto, não são lineares. O aumento do tamanho do território potencializa tanto as suas vantagens quanto seus custos; o ponto em que essas duas forças se equilibram é onde o território tem seu tamanho ótimo (MacLean \& Seastedt 1978).

Segundo o modelo de defesa territorial ótima de López-Sepulcre \& Kokko (2005), tanto altas taxas reprodutivas quanto custos elevados na defesa territorial levam a territórios pequenos. A explicação para o alto custo da defesa territorial levar à existência de territórios pequenos é intuitiva. Já no caso das taxas reprodutivas, não, principalmente porque o aumento da capacidade reprodutiva, como já dito, é um dos benefícios esperados pela aquisição de territórios maiores. Entretanto, outra conseqüência da alta capacidade reprodutiva é o rápido crescimento da população, que leva ao aumento da competição pelos territórios, incrementando os custos da defesa territorial e, por fim, constringindo o tamanho dos territórios. Inversamente, populações que apresentam baixas taxas reprodutivas e baixos custos na defesa territorial, possuem territórios grandes.

\section{IMPLICAÇÕES PARA A CONSERVAÇÃO}

A territorialidade é frequentemente utilizada como uma ferramenta para estimativas de densidade populacional. Combinada com modelos de disponibilidade de habitat, a densidade permite estimar o número de indivíduos em uma população, o que é necessário para definir o status de ameaça de uma espécie segundo os critérios da IUCN.

Osindivíduosflutuantes sãode extremaimportância para a conservação, não apenas por representarem uma fração significativa da população (Kokko et al. 2001), como também de sua variabilidade genética, além de serem os responsáveis pelo tamponamento no número de pares reprodutores (Newton 1992). Por este motivo, o impacto da perda de habitat possivelmente é mais intenso na fração flutuante da população do que na fração reprodutora. A prática de documentar os declínios populacionais apenas com o registro dos pares reprodutores pode subestimar o real declínio (Hunt 1998, Kokko et al. 2001).

Por outro lado, os flutuantes podem representar um grande risco para espécies ameaçadas e/ou populações em declínio. Emblemático é o exemplo da espécie de Muscicapidae (família dos sabiás) Copsychus sechellarum endêmica das Ilhas Seichelles, que em 1989 era representada por uma população de cerca de 20 indivíduos. Destes, cinco tornaram-se flutuantes em habitats ótimos, levando o sucesso reprodutivo de quatro casais a zero naquele ano (Komdeur 1996). Programas de conservação que incluem a suplementação alimentar também podem gerar uma redução do sucesso reprodutivo. A oferta extra de alimento atrai os flutuantes, que vão interferir na reprodução dos pares que possuem território (Carrete et al. 2006). Portanto, é importante considerar a atuação de indivíduos flutuantes ao estabelecer estratégias de conservação.

Em síntese, o comportamento territorial, apesar de ser um tema importante para a ecologia de aves (particularmente ecologia de populações e conservação), ainda apresenta vários aspectos que necessitam de um maior conhecimento e merecem a atenção dos pesquisadores. Padrões gerais indicam a territorialidade como um dos fatores reguladores da densidade de populações, sendo também recorrente a associação positiva entre sucesso reprodutivo e qualidade de território. Por outro lado, a interação de donos de 
territórios com flutuantes, e suas implicações em nível individual e populacional, ainda é controversa.

AGRADECIMENTOS: Aos revisores anônimos; à Regina Macedo, Carlos Eduardo Grelle e Fernando Fenandez pelas contribuições ao manuscrito, à CAPES pela bolsa de Mestrado concedida à VCT e ao CNPq pela bolsa de Produtividade em Pesquisa e auxílio de pesquisa associado concedidos à MASA (processo no 3027185/03-6).

\section{REFERÊNCIAS BIBLIOGRÁFICAS}

ADAMS, E.S. 2001. Approaches to the study of territory size and shape. Annual Review of Ecology and Systematics, 32: 277-303.

ALEXANDER, W.B. 1936. "Territory" recorded for nightingale in seventeenth century. British Birds, 29: 388.

ALLEN, A.A. 1934. Sex rhythm in the ruffed grouse (Bonasa umbellus Linn.) and other birds. The Auk, 51: 180-199.

ARCESE, P.; SMITH, J.N.M; HOCHACHKA, W.M.; ROGERS, C.M. \& LUDWIG, D. 1992. Stability, regulation, and the determination of abundance in an insular song sparrow population. Ecology, 73: 805-822.

BARG, J.J.; JONES, J. \& ROBERTSON, R.J. 2005. Describing breeding territories of migratory passerines: suggestions for sampling, choice of estimator, and delineation of core areas. Journal of Animal Ecology, 74: 139-149.

BIBBY, C.J. 2003. Fifty years of bird study. Bird Study, 50: 194-210.

BLONDEL, J.; PERRET, P. \& GALAN, M.J. 2000. High divorce rates in Corsican blue tits: how to choose a better option in a harsh environment. Oikos, 89: 451-460.

BOCK, W.J. 2001. Contributions of Central European ornithology to world ornithology. Journal of Ornithology, 1: 94-108.

BOTH, C. \& VISSER, M.E. 2000. Breeding territory size affects fitness: an experimental study on competition at the individual level. Journal of Animal Ecology, 69: 1021-1030.

BROWN, J.L. 1964. The evolution of diversity in avian territorial systems. The Wilson Bulletin, 76: 160-169.

BROWN, J.L. 1969. Territorial behavior and population regulation in birds - a review and re-evaluation. The Wilson Bulletin, 1: 293-329.

BRUINZEEL, L.W. \& VAN DE POL, M. 2004. Site attachment of floaters predicts success in territory acquisition. Behavioral Ecology, 15: 290-296.

BRUSH, T. 1983. Cavity use by secondary cavity-nesting birds and response to manipulations. The Condor, 85: 461-466.
CARRETE, M.; DONÁZAR, J.A. \& MARGALIDA, A. 2006. Density-dependent productivity depression in pyrenean bearded vultures: implications for conservation. Ecological Applications, 16: 1674-1682.

DAVIES, D.E. 1941. The relation of abundance to territorialism in tropical birds. Bird-Banding 12: 93-97.

DAVIES, N.B. 1992. Dunnock behaviour and social evolution. Oxford University Press, Oxford. 290p.

DAVIES, N.B \& HOUSTON, A.I. 1981. Owners and satellites: the economics of territory defence in the pied wagtail, Motacilla alba. Journal of Animal Ecology, 50: 157-180.

DICKINSON, J.L. 1997. Male detention affects extra-pair copulation frequency and pair behavior in western bluebirds. Animal Behaviour, 53: 561-571.

DUNN, E.H. \& TESSAGLIA, D.L. 1994. Predation of birds at feeders in winter. Journal of Field Ornithology, 65: 8-16.

FORT, K.T. \& OTTER, K.A. 2004. Territorial breakdown of black-capped chickadees, Poecile atricapillus, in disturbed habitats? Animal Behaviour, 68: 407-415.

GIBB, J. 1956. Food, feeding habitats and territory of the rock pipit Anthus spinoletta. Ibis, 98: 506-530.

GILL, F.B. \& WOLF, L.L. 1975. Economics of feeding territoriality in the golden-winged sunbird. Ecology, 56(2): 333-345.

GILROY, J.J. \&SUTHERLAND, W.J. Beyond ecological traps: perceptual errors and undervalued resources. Trends in Ecology and Evolution, 22: 351-356.

GRAFEN, A. 1987. The logic of divisively asymmetric contests: respect for ownership and the desperado effect. Animal Behaviour, 35: 462-467.

HAHN, S. \& BAUER, S. 2008. Dominance in feeding territories relates to foraging success and offspring growth in brown skuas Catharacta antartica lonnbergi. Behavioral Ecology and Sociobiology, 62: 1149-1157.

HEG, D.; BRUIZEEL, L.W \& ENS, B.J. 2003. Fitness consequences of divorce in the oystercatcher, Haemantopus ostralegus. Animal Behaviour, 66: 175-184.

HEG, D.; ENS, B.J.; VAN DER JEUGD, H.P. \& BRUINZEELl, L.W. 2000. Local dominance and territorial settlement of nonbreeding oystercatchers. Behaviour, 137: 473-530.

HINDE, A. 1956. The biological significance of the territories of birds. Ibis, 98: 340-369.

HOWARD, H.E. 1920. Territory in bird life. Murray, London. 308p. 
HUNT, W.G. 1998. Raptor floaters at Moffat's equilibrium. Oikos, 82: 191-197.

ITO, Y. 1980. Comparative ecology. Cambridge University Press, Cambridge. 448p.

JENKINS, J.M. \& JACKMAN, R.E. 1993. Mate and nest-site fidelity in a resident population of bald eagles. The Condor, 95: 1053-1056.

JOHNSON, L.S.; KERMOTT, L.H. \& LEIN, M.R. 1994. Territorial polygyny in house wrens: are females sufficiently compensated for the cost of mate sharing? Behavioral Ecology, 5: 98-104.

KEMPENAERS, B.; EVERDING, S.; BISHOP, C.; BOAG, P. \& ROBERTSON, R.J. 2001. Extra-pair paternity and the reproductive role of male floaters in the tree swallow (Tachycineta bicolor). Behavioral Ecology and Sociobiology, 49: 251-259.

KOKKO, H.; LÓPEZ-SEPULCRE, A. \& MORREL, L.J. 2006. From hawks and doves to self-consistent games of territorial behavior. The American Naturalist, 167: 901-912.

KOKKO, H. \& SUTHERLAND, W.J. 1998. Optimal floating and queuing strategies: consequence for density dependence and habitat loss. The American Naturalist, 152: 354-366.

KOKKO, H.; SUTHERLAND, W.J. \& JOHNSTONE, R.A. 2001. The logic of territory choice: implications for conservation and source-sink dynamics. The American Naturalist, 157: 459-463.

KOMDEUR, J. 1996. Breeding of the Seychelles magpie robin Copsychus sechellarum and implications for its conservation. Ibis, 138: 485-498.

LACK, D. 1954. Two robin population. Bird Study, 1: 14-17.

LEFFLAAR, D. \& ROBERTSON, R.J. 1984. Do male tree swallows guard their mates? Behavioral Ecology and Sociobiology, 16: 73-79.

LOPEZ-SEPULCRE, A. \& KOKKO, H. 2005. Territorial defence, territory size, and population regulation. The American Naturalist, 166: 317-329.

MACLEAN, S.F. \& SEASTEDT, T.R. 1978. Avian territoriality: sufficient resources or interference competition. The American Naturalist, 114: 308-312.

MAHER, C.R. \& LOTT, D.F. 1995. Definitions of territoriality used in the study of variation in vertebrate spacing systems. Animal Behaviour, 49: 1581-1597.

MAYR, E. 1935. Bernard Altum and the territory theory. Proceedings of the Linnean Society of New York, 45-46: 24-30.
MØLLER, A.P. \& NINNI, P. 1998. Sperm competition and sexual selection: a meta-analysis of paternity studies of birds. Behavioral Ecology and Sociobiology, 43: 345-358.

MYERS, J.P.; CONNORS, P.G. \& PITELKA, F.A. 1979. Territory size in wintering sanderlings: effects of prey abundance and intruder density. The Auk, 96: 551-561.

NEWTON, I. 1992. Experiments on the limitation of bird numbers by territorial behavior. Biological Reviews, 67: 129-173.

NICE, M.M. 1941. The role of territory in bird life. American Midland Naturalist, 26: 441-487.

PARADIS, E.; BAILLIE， S.R.; SUTHERLAND, W.J.; GREGORY, R.D. 2002. Exploring density-dependent relationships in demographic parameters in populations of birds at large spatial scale. Oikos, 97: 293-307.

PEN, I. \& WEISSING, F.J. 2000. Optimal floating and queuing strategies: the logic of territory choice. The American Naturalist, 155: 512-526.

POESEL, A.; DABELSTEEN, T.; DARDEN, S.K.; DELHEY, K. \& PETERS, A. 2007. Territorial responses of male blue tits, Cyanistes caeruleus, to UV-manipulated neighbours. Journal of Ornithology, 148: 179-187.

PRYKE, S.R. \& ANDERSSON, S. 2003. Carotenoid-based status signaling in red-shouldered widowbirds (Euplectes axillaris): epaulet size and redness affect captive and territorial competition. Behavioral Ecology and Sociobiology, 53: 393-401.

SANDELL, M.I. \& DIEMER, M. 1999. Intraspeceific brood parasitism: a strategy for floating females in the european starling. Animal Behaviour, 57: 197-202.

SIH, A. \& MATEO, J. 2001. Punishment and persistence pay: a new model of territory establishment and space use. Trends in Ecology and Evolution, 16: 447-449.

VAN WINKLE, W. 1975. Comparison of several probabilistic home-range models. Journal of Wildlife Management, 39: 118-123.

VICKERY, P.D.; HUNTER, M.L. \& WELLS, J.V. Is density an indicator of breeding success? The Auk, 109: 706-710.

Submetido em 17/09/2008. Aceito em 04/12/2008. 